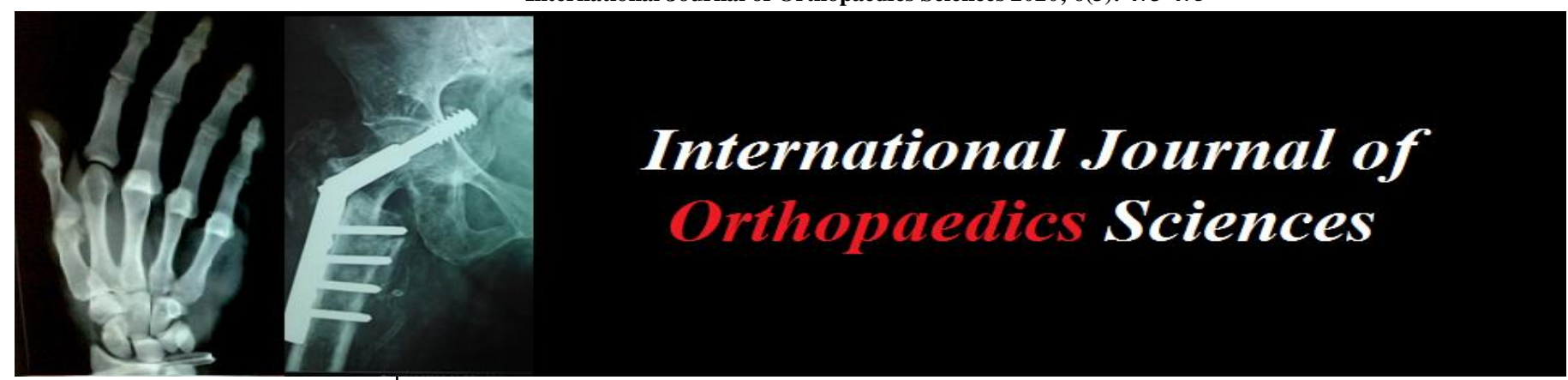

E-ISSN: 2395-1958

P-ISSN: 2706-6630

IJOS 2020; 6(3): 473-475

(C) $2020 \mathrm{IJOS}$

www.orthopaper.com

Received: 04-05-2020

Accepted: 06-06-2020

Ahmad Alriyaee

Orthopedic Resident, MBBS,

King Salman Military Hospital,

Saudi Arabia

MHD Hamam Alkhatib

Intern medical student

Near east university

Northern Ciprus, Cyprus

Dr. Yassin Mustafa

Radiology Consultant, MBBS,

King Salman Military Hospital,

Saudi Arabia

Dr. Ahmad Awaji

Arthroplasty and Lower

Extremity Reconstruction

Surgeon, MBBS, King Salman

Military Hospital, Saudi Arabia

Corresponding Author:

Ahmad Alriyaee

Orthopedic Resident, MBBS,

King Salman Military Hospital,

Saudi Arabia

\section{The impact of teardrop sign on DDH prognosis}

\author{
Ahmad Alriyaee, MHD Hamam Alkhatib, Yassin Mustafa and Ahmad \\ Awaji
}

DOI: https://doi.org/10.22271/ortho.2020.v6.i3h.2238

Abstract

Introduction: DDH is a common developmental dysplasia in children. The acetabular teardrop sign is an important tool and has not become established due to the relative subjectivity.

Objectives: To study the shape of acetabular teardrop sign and its changes after reduction at initial diagnosis, 6 months, one, and 2 years after reduction, its width, shape, and types of the teardrop, the thickness of the acetabular floor, and the center edge angle measured of dislocated and contralateral normal hips. The teardrops figures were classified into 4 distinct groups: absent, v-shaped, u shaped, and inverted or crossed shape.

Methods: a retrospective cohort study of one sixty-four patients involved in the study which took place in King Salman military hospital, Tabuk, $90.4 \%$ of them are girls and $9.6 \%$ are boys.

Results: there is a statistically significant impact of superior width, acetabular thickness of teardrop sign after reduction at $6,12,18,24$ months, there is a statistically significant impact of inferior width only at 2 years, and there are no significant changes of the shape of teardrop sign after reduction.

Keywords: Acetabular index, central edge angle, DDH, hip, teardrop sign

\section{Introduction}

Developmental dysplasia of the hip (DDH) is a disorder of abnormal development resulting in dysplasia, subluxation, and possible dislocation of the hip secondary to capsular laxity and mechanical factors. Treatment varies from Pavlik bracing to surgical reduction and osteotomies depending on the age of the patient and the degree of dysplasia. There are different tools for the measurement of dislocated and reduced hips either clinically and radiographically ${ }^{[5]}$. The acetabular teardrop is an important tool for the diagnosis of acetabular dysplasia. Since it was first described by Köhler its widespread use has not become established due to the relative subjectivity in the classification of the types, shape, and their variance after good reduction achieved ${ }^{[4]}$. The significance of teardrop changes in DDH patients is thought to be the prediction of acetabular development and changes after reduction in the treatment of DDH is early and there will be a further impact on further management if need it because it would help ensure optimal timing of additional procedure if necessary.

A few studies have been found focusing on this topic. The researchers have reviewed most of those studies focusing on this topic and it found very rare research on it. There was no relationship between the type of teardrop and the final results. The continued presence of a Vshaped teardrop, widening of the superior width of the teardrop, and thickening of the acetabular floor are suggestive of residual dysplasia ${ }^{[1]}$. Other studies talk about the appearance of the acetabular teardrop within 6 months after the reduction of the hip is strongly associated with a favorable long-term outcome. We believe that the appearance of the teardrop is the earliest radiographic sign that a stable, concentric reduction of the hip has been achieved [2]. And other study talks about we retrospectively reviewed the radiographs of the pelvis and hips of forty-five patients who had unilateral congenital dislocation of the hip treated with closed reduction and application of a cast without subsequent operations. The radiographs were made at the time of the initial diagnosis, two years after the reduction, when the child was ten years old, and at skeletal maturity. The width, shape, and type of the teardrop; the thickness of the acetabular floor; the acetabular index; the center-edge angle; the articulo-trochanteric distance; and the Severin class at maturity were measured in the dislocated and contralateral normal hips. 
At the time of the initial diagnosis, a well-defined teardrop was seen in thirty-six (80 percent) of the normal hips and seven (16 percent) of the dislocated hips. There was no difference in the width of the teardrop in the seven dislocated hips compared with that in the normal hips, although the Vshaped and crossed types of teardrops were more frequent in the dislocated hips. The $\mathrm{V}$ shape was not observed in the normal hips but was seen in sixteen dislocated hips two years after the reduction and in twelve dislocated hips when the children were ten years old. The superior and inferior widths of the teardrop of the dislocated hips were significantly greater than those of the normal hips $(p<0.001$ and $p<0.05$, respectively) when the children were ten years old. The hips with residual acetabular dysplasia had a V-shaped teardrop, widening of the superior width of the teardrop, and thickening of the acetabular floor. These hips, which were usually Sever in class IV at the time of skeletal maturity, had a poor prognosis in adult life ${ }^{[3]}$.

\section{Methodology}

The study used a cross-sectional retrospective cohort descriptive design and was conducted in Tabuk, KSA. The setting was at king Salman military hospital clinics. Data collection started on 11 April, 2019, and was completed within 6 months. The study included DDH patients in Tabuk, from different nationalities, genders, and their age range between 6 and 77 months. The result shows that $46.9 \%$ of the sample age ranges between 13 to 24 months, $25 \%$ were above 24 months, $18.8 \%$ were between 7 to 13 months, while $9.4 \%$ were less than 7 months. Participants were DDH children unilateral involvement and treated with a single successful attempt of reduction, closed, open, with or without osteotomy. There was an attempt to have proper $\mathrm{x}$ rays views to get precise angels measurement and shapes of teardrop sign-on pelvic x-ray at a different time of the study and exclude unclear samples.

Table 1: Correlation coefficients for variables

\begin{tabular}{|c|c|c|c|c|}
\hline Variables & At Presentation & 6 months & 1 year & 2 years \\
\hline superior width of affected side & 0.356 & 0.734 & 0.672 & 0.699 \\
\hline superior width of non-affected side & 0.159 & -0.030 & 0.101 & 0.273 \\
\hline inferior width of affected side & -0.308 & 0.188 & 0.276 & 0.756 \\
\hline inferior width of non-affected side & 0.207 & -0.022 & -0.063 & 0.537 \\
\hline acetabular thickness of affected & 0.582 & 0.778 & 0.585 & 0.523 \\
\hline acetabular thickness of non-affected side & 0.477 & 0.516 & 0.505 & 0.516 \\
\hline teardrop figure of affected side & -0.017 & 0.081 & -0.174 & -0.113 \\
\hline teardrop figure of non-affected side & 0.327 & 0.520 & 0.340 & 0.449 \\
\hline
\end{tabular}

\subsection{Data sources/measurements}

The data have been collected by assessing and collecting the result of the radiographs which have been obtained at the time of initial diagnosis, 6 months, one, and 2 years after reduction, the width, shape, and type of teardrop, the thickness of the acetabular floor, and the center edge angel were measures of dislocated and contralateral normal hips. The teardrops figures were classified into 4 distinct groups: absent, Vshaped, U-shaped, and inverted or crossed shape. The result of treatment was classified as a satisfactory group: CE angel $>20$, and unsatisfactory group: CE angel $<20$ Judged by CE at final follow up.

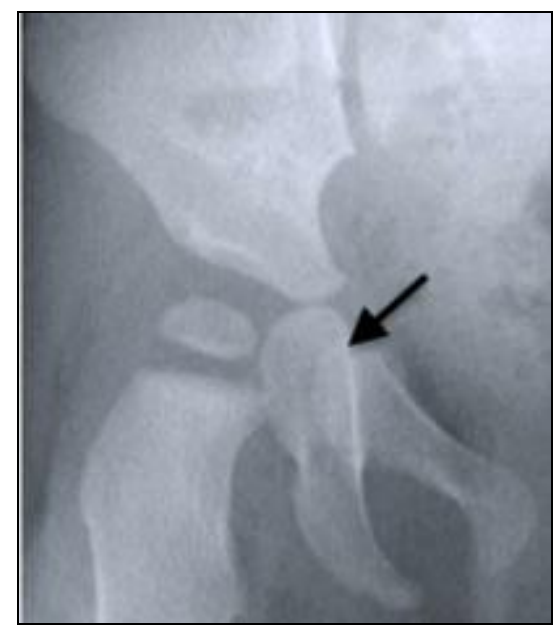

Fig 1: X-ray for one of the participants 6 months post-reduction

\subsection{Statistical analysis}

The raw data was processed and cleaned before the statistical analysis. Data management and analyses were carried out by using the IBM Statistical Software for Social Sciences (SPSS) for Windows, version 25 (IBM Corp., Armonk, N.Y., USA).
Qualitative variables are presented as frequency and percentage and the quantitative variables as mean and standard deviation. The data comparison was carried out with a p-value of 0.05 .

\section{Results}

The sample size was 64 patients. The majority of participants $(90.4 \%)$ were female. In terms of age, the majority (46.9\%) their age between 13 and 24 months old, with $25 \%$ above 24 months and $18.8 \%$ their age between 7 and 13 months old, while only $9.4 \%$ their age less than 7 months. The result shows that $65.6 \%$ of the sample is affected on the left side, while $34.4 \%$ are affected on the right side. As per the superior width of teardrop sign, there was a statistically significant effect of the superior width of the affected side 6 months 1-2 years post reduction and the superior width of non-affected side 6 months, 1-2 years post-reduction on central edge angel of non-affected side 6 months, 1-2 years post-reduction. while was a statistically significant effect of the superior width of affected side 6 months, 1-2 years post-reduction on central edge angel of affected side 6 months, 1-2 years post-reduction but was not a statistically significant effect of the superior width of non-affected side 6 months, 1-2 years post-reduction on the central edge angel of affected side 6 months, 1-2 years post-reduction. As per inferior width of teardrop sign, there was a statistically significant effect of the inferior width of affected side 2 years post-reduction on the central edge angel of affected side 2 years post-reduction but was not a statistically significant effect of the inferior width of affected side 6 months, 1-year post-reduction on the central edge angel of affected side 6 months, 1-year post-reduction. As per the non-affected side, there was no statistically significant effect of the inferior width of the non-affected side 6 months 1,2 years post-reduction on the central edge angel of non-affected side 6 months 1,2 years post-reduction. 
As per acetabular thickness, there was a statistically significant effect of acetabular thickness of affected side 6 months 1, 2 years post-reduction on the central edge angel of affected side 6 months 1, 2 years post-reduction but there was no statistically significant effect of the acetabular thickness of non-affected side 6 months 1,2 years post-reduction on the central edge angle of non-affected side 1-year post-reduction. As per teardrop figure, there was a no statistically significant effect of the teardrop figure of affected side 6 months 1,2 years post-reduction on the central edge angel of affected side 6 months 1, 2 years post-reduction. But it was a statistically significant effect of the teardrop figure of non-affected side 6 months 1, 2 years post-reduction on the central edge angel of non-affected side 6 months 1, 2 years post-reduction.

\section{Discussion}

The significance of teardrop changes in DDH patients thought to be the prediction of acetabular development and changes after reduction in the treatment of DDH eerily. Since it was first described by Köhler its widespread use has not become established due to the relative subjectivity in the classification of the types, shape, and their variance after good reduction achieved ${ }_{[4]}$. The development and growth of the teardrop sign still not fully understood. In this paper, it is expected to get more clues to see how the teardrop sign grows with the time after reduction like more growth in superior width and acetabular thickness and decrease in inferior width and change of the shape of teardrop from X or V shape to U shape after reduction. The superior width of teardrop sign shows significant impact after reduction as it was increased after 6 , 12,18 , and 24 months on both affected side and non-affected side which could be attributed to the development of the pelvis and hip as it grows and increases in both affected and non-affected side. The inferior width of teardrop show statically significant 2 years post-reduction but there was no significant effect after $6,12,18$ months on the affected side only which is maybe related to the growth of the teardrop sign in the pelvis after reduction and the pelvis configuration get the normal sense of reduced hip.

As per an acetabular thickness, there was a statistically significant effect of acetabular thickness of affected side 6 months 1, 2 years post-reduction on the central edge angel of affected side 6 months 1, 2 years post-reduction but was not a statistically significant effect of the acetabular thickness of non-affected side 6 months 1, 2 years post-reduction on the central edge angel of non-affected side 1-year post-reduction, so the acetabular thickness after reduction differs and has a significant impact after reduction on the configuration of acetabulum signs. As per teardrop figure, there was a was not a statistically significant effect of the teardrop figure of affected side 6 months 1, 2 years post-reduction on the central edge angel of affected side 6 months 1, 2 years postreduction. But it was a statistically significant effect of the teardrop figure of non-affected side 6 months 1,2 years postreduction on the central edge angel of non-affected side 6 months 1, 2 years post-reduction. The shape of teardrop (figure) may take a long time to change in its configuration.

\section{Conclusion}

$\mathrm{DDH}$ is a common developmental dysplasia in children, diagnosis modalities wide. The acetabular teardrop sign is an important tool and has not become established due to the relative subjectivity. There are changes in the developmental of teardrop sign of normal and dislocated hip which is proved in this research but it's not fully understood.

\section{References}

1. Sibiński M, Kozlowski P, Synder M. Ocena kształtowania lzy köhlera po bezoperacyjnym leczeniu rozwojowej dysplazji stawu biodrowego [The teardrop development after conservative treatment of developmental dysplasia of the hip]. Chir Narzadow Ruchu Ortop Pol. 2005; 70(3):173-177.

2. Smith JT, Matan A, Coleman SS, Stevens PM, Scott SM. The predictive value of the development of the acetabular teardrop figure in developmental dysplasia of the hip. J Pediatr Orthop. 1997; 17(2):165-169. Doi: 10.1097/00004694-199703000-00005

3. Erkula G, Celikbas E, Kilic BA, Demirkan F, Kiter AE. The acetabular teardrop and ultrasonography of the hip. J Pediatr Orthop B. 2004; 13(1):15-20. Doi: 10.1097/00009957-200401000-00003

4. Albiñana J, Morcuende JA, Weinstein SL. The teardrop in congenital dislocation of the hip diagnosed late. A quantitative study. J Bone Joint Surg Am. 1996; 78(7):1048-1055.

Doi: 10.2106/00004623-199607000-00009 